\title{
SOME CHARACTERIZATIONS OF THE EULER GAMMA FUNCTION
}

\author{
JANUSZ MATKOWSKI
}

\begin{abstract}
Assume that $f:(0, \infty) \rightarrow(0, \infty)$ is bounded from above on a set of positive Lebesgue measures or on a set of the second category with the Baire property and satisfies the functional equation $f(x+1)=x f(x)$ for $x>0$ and $f(1)=1$. We prove that, if there is a positive sequence $\left(p_{n}\right), \lim _{n \rightarrow \infty} p_{n}=\infty$, such that for every $n \in \mathbb{N}$, the function $x \mapsto \log \left(x^{p_{n}}\right)$ is Jensen convex in the interval $(1, \infty)$; or there are two positive sequences $\left(p_{n}\right)$ and $\left(q_{n}\right)$, $\lim _{n \rightarrow \infty} p_{n}=\infty, \lim _{n \rightarrow \infty} q_{n}=0$ such that, for every $n \in \mathbb{N}$, the function $x \mapsto\left[f\left(x^{p_{n}}\right)\right]^{q_{n}}$ is Jensen convex in the interval $(1, \infty)$, then $f$ is the Euler gamma function.
\end{abstract}

1. Introduction. In 1922, Bohr and Mollerup [3] proved that if a function $f:(0, \infty) \rightarrow(0, \infty)$ satisfies the functional equation

$$
f(x+1)=x f(x), \quad x>0 ; f(1)=1,
$$

and $\log \circ f$ is convex, then $f$ is the Euler gamma function $\Gamma$ (cf., also Artin [2]).

Gronau and Matkowski [4] in 1993 gave an improvement of this result, showing, in particular, that (under weak regularity of $f$ ) it remains true if the convexity of $\log \circ f$ is replaced by the much weaker condition of the geometrical convexity of $f$ in some interval $(b, \infty)$, that is,

$$
f(\sqrt{x y}) \leq \sqrt{f(x) f(y)}, \quad x, y>b,
$$

and equivalent to the Jensen convexity of the function log $\circ f \circ$ exp.

In a recent paper, Alzer and Matkowski [1] have obtained a characterization of the Gamma function, making use of some properties of the composition of the power functions with the function $\Gamma \circ$ exp which

2010 AMS Mathematics subject classification. Primary 26A51, 26D07, 33B15, 39B22.

Keywords and phrases. Gamma function, convex function, geometrically convex function, functional equation, characterization.

Received by the editors on March 11, 2013, and in revised form on July 19, 2013. DOI:10.1216/RMJ-2015-45-4-1225 
reads as follows. Assume that $f:(0, \infty) \rightarrow(0, \infty)$ satisfies (1). If $f$ is bounded on a set of positive Lebesgue measure (or on a set of the second category with the Baire property) and there are $a>0$ and a sequence of positive numbers $q_{n}$ with $\lim _{n \rightarrow \infty} q_{n}=0$ such that, for every $n$ the function $(f \circ \exp )^{q_{n}}$ is Jensen convex, then $f$ is the gamma function.

The characterization of the gamma function presented in this note is also based on equation (1). The main result, Theorem 2 in Section 3, reads as follows. Assume that $f:(0, \infty) \rightarrow(0, \infty)$ is bounded from above on a set of positive Lebesgue measures or on a set of the second Baire category with the Baire property and satisfies the functional equation (1). If there is a positive sequence $\left(p_{n}\right), \lim _{n \rightarrow \infty} p_{n}=\infty$ such that, for every $n \in \mathbb{N}$, the function $x \mapsto \log f\left(x^{p_{n}}\right)$ is Jensen convex in the interval $(1, \infty)$; or there are two positive sequences $\left(p_{n}\right)$ and $\left(q_{n}\right), \lim _{n \rightarrow \infty} p_{n}=\infty, \lim _{n \rightarrow \infty} q_{n}=0$ such that, for every $n \in \mathbb{N}$, the function $x \mapsto\left[f\left(x^{p_{n}}\right)\right]^{q_{n}}$ is Jensen convex in the interval $(1, \infty)$, then $f$ is the Euler gamma function. In Section 1 we present a simple argument assuming that $f$ is a twice differentiable function (Theorem 1). In Section 2, we present the counterparts of these results under a little stronger assumption that can be regarded as a motivation of the main results.

As an immediate corollary, we obtain the following. If $f:(0, \infty) \rightarrow$ $(0, \infty)$ satisfies (1) and, for every positive integer $n$, the function $x \mapsto\left[f\left(x^{n}\right)\right]^{1 / n}$ is convex, then $f$ is the Gamma function.

2. A characterization for regular functions. Let us note that the following is easy to verify

Remark 2.1. Let $I \subset(0, \infty)$ be an open interval, and let $f: I \rightarrow$ $(0, \infty)$ be twice differentiable. The following conditions are pairwise equivalent:

(i) the function $f$ is geometrically convex, that is,

$$
f\left(x^{t} y^{1-t}\right) \leq f(x)^{t} f(y)^{1-t}, \quad x, y \in I, t \in(0,1) ;
$$

(ii) the function $\log \circ f \circ \exp$ is convex in the interval $J:=\log (I)$;

(iii) the function $f: I \rightarrow(0, \infty)$ satisfies the inequality

$$
f(x) f^{\prime \prime}(x) x+f(x) f^{\prime}(x) \geq\left[f^{\prime}(x)\right]^{2} x, \quad x \in I .
$$


We prove the following:

Theorem 2.2. Suppose that a function $f:(0, \infty) \rightarrow(0, \infty)$ is twice differentiable and satisfies equation (1). If $f$ satisfies one of the following two conditions:

(i) there is a sequence $\left(p_{n}\right), p_{n} \rightarrow \infty$, such that for every $n \in \mathbb{N}$, the function $x \mapsto \log f\left(x^{p_{n}}\right)$ is convex in $(1, \infty)$;

(ii) there exist some sequences of positive numbers $\left(p_{n}\right),\left(q_{n}\right) ; p_{n} \rightarrow$ $\infty, q_{n} \rightarrow 0$, such that, for every $n \in \mathbb{N}$, the function $x \mapsto$ $\left[f\left(x^{q_{n}}\right)\right]^{p_{x}}$ is convex in $(1, \infty)$,

then $f$ is the Euler gamma function.

Proof. To prove the first result, take $p>0$. Since $f$ is twice differentiable, the function $x \mapsto \log f\left(x^{p}\right)$ is convex in $(1, \infty)$ if, and only if,

$$
\begin{aligned}
\left(\log f\left(x^{p}\right)\right)^{\prime \prime}= & \frac{p^{2} x^{p-2}}{\left[f\left(x^{p}\right)\right]^{2}}\left\{\frac{p-1}{p} f\left(x^{p}\right) f^{\prime}\left(x^{p}\right)\right. \\
& \left.+x^{p} f\left(x^{p}\right) f^{\prime \prime}\left(x^{p}\right)-x^{p}\left[f^{\prime}\left(x^{p}\right)\right]^{2}\right\} \geq 0,
\end{aligned}
$$

for all $x \in(1, \infty)$. Since, for $p>0$, the function $x \mapsto x^{p}$ maps the interval $(1, \infty)$ onto itself, this inequality is satisfied if, and only if,

$$
\frac{p-1}{p} f(x) f^{\prime}(x)+x f(x) f^{\prime \prime}(x)-x\left[f^{\prime}(x)\right]^{2} \geq 0, \quad x \in(1, \infty) .
$$

Replacing here $p$ by $p_{n}$ such that $p_{n} \rightarrow \infty$, and then letting $n \rightarrow \infty$, we obtain

$$
f(x) f^{\prime}(x)+x f(x) f^{\prime \prime}(x)-x\left[f^{\prime}(x)\right]^{2} \geq 0, \quad x \in(1, \infty) .
$$

In view of Remark 2.1 , the function $f$ is geometrically convex in $(1, \infty)$. Since $f$ satisfies (1), in view of the Gonau-Matkowski result [4], the function $f$ must be the Euler gamma function.

To prove the second result take arbitrary positive real numbers $p$ and $q$. The function $x \mapsto\left[f\left(x^{p}\right)\right]^{q}$ is convex in the interval $(1, \infty)$ if, 
and only if,

$$
\begin{aligned}
& \frac{\left(\left[f\left(x^{p}\right)\right]^{q}\right)^{\prime \prime}}{p^{2} q x^{p-2}\left[f\left(x^{p}\right)\right]^{q-2}} \\
= & (q-1) x^{p}\left[f^{\prime}\left(x^{p}\right)\right]^{2}+x^{p} f\left(x^{p}\right) f^{\prime \prime}\left(x^{p}\right)+\frac{p-1}{p} f\left(x^{p}\right) f^{\prime}\left(x^{p}\right) \geq 0,
\end{aligned}
$$

for all $x \in(1, \infty)$. Since $p$ and $q$ are positive, and the function $x \mapsto x^{p}$ maps the interval $(1, \infty)$ onto itself, we see that this inequality is satisfied if, and only if,

$$
(q-1) x\left[f^{\prime}(x)\right]^{2}+x f(x) f^{\prime \prime}(x)+\frac{p-1}{p} f(x) f^{\prime}(x) \geq 0, \quad x \in(1, \infty) .
$$

Setting here $p=p_{n}, q=q_{n}, p_{n} \rightarrow \infty$ and $q_{n} \rightarrow 0$, and letting $n \rightarrow \infty$, we obtain

$$
-x\left[f^{\prime}(x)\right]^{2}+x f(x) f^{\prime \prime}(x)+f(x) f^{\prime}(x) \geq 0, \quad x \in(1, \infty),
$$

whence, by Remark 2.1, the function $f$ is geometrically convex. Now the result follows from the main result of [4].

3. Main results. Let $D \subset \mathbb{R}^{k}$ be convex and open, and let $A \subset D$ be of positive Lebesgue measure. We need the following result of Ostrowski [8] (see also [6, page 210]).

If $f: D \rightarrow R$ is Jensen convex, that is,

$$
f\left(\frac{x+y}{2}\right) \leq \frac{f(x)+f(y)}{2}, \quad x, y \in D,
$$

and bounded from above on $A$, then $f$ is convex.

A subset $A$ of a topological space $X$ is said to have the Baire property if $A=(D \cup P) \backslash R$, where the set $D$ is open, and the sets $P, R$ are of the first category. (The family of sets having the Baire property is a $\sigma$-algebra.)

Let $D \subset \mathbb{R}^{k}$ be convex and open, and let $A \subset D$ be of the second category with the Baire property. We shall also need the following result, which is due to Mehdi [7] (see also [6, page 210]).

If $f: D \rightarrow R$ is Jensen convex and bounded from above on $A$, then $f$ is convex. 
The main result of the present paper reads as follows:

Theorem 3.1. Assume that $f:(0, \infty) \rightarrow(0, \infty)$ is bounded from above on a set of positive Lebesgue measure or on a set of the second category with Baire property, and satisfies equation (1):

$$
f(x+1)=x f(x), \quad x>0 ; f(1)=1 .
$$

If one of the following two conditions is fulfilled,

(i) there is a positive sequence $\left(p_{n}\right), \lim _{n \rightarrow \infty} p_{n}=\infty$ such that, for every $n \in \mathbb{N}$, the function

$$
x \longmapsto \log f\left(x^{p_{n}}\right)
$$

is Jensen convex in the interval $(1, \infty)$;

(ii) there are two positive sequences $\left(p_{n}\right)$ and $\left(q_{n}\right), \lim _{n \rightarrow \infty} p_{n}=\infty$, $\lim _{n \rightarrow \infty} q_{n}=0$ such that, for every $n \in \mathbb{N}$, the function

$$
x \longmapsto\left[f\left(x^{p_{n}}\right)\right]^{q_{n}}
$$

is Jensen convex in the interval $(1, \infty)$,

then $f$ is the Euler gamma function.

Proof. If condition (i) is satisfied, then

$$
\log f\left(\left(\frac{x+y}{2}\right)^{p_{n}}\right) \leq \frac{\log f\left(x^{p_{n}}\right)+\log f\left(y^{p_{n}}\right)}{2}, \quad x, y>1 ; n \in \mathbb{N},
$$

whence, replacing $x$ and $y$, respectively, by $x^{1 / p_{n}}$ and $y^{1 / p_{n}}$, we obtain

$$
f\left(\left(\frac{x^{1 / p_{n}}+y^{1 / p_{n}}}{2}\right)^{p_{n}}\right) \leq \sqrt{f(x) f(y)}, \quad x, y>1 ; n \in \mathbb{N} .
$$

Since

$$
\lim _{r \rightarrow \infty}\left(\frac{u^{1 / r}+v^{1 / r}}{2}\right)^{r}=\sqrt{u v}, \quad u, v>0,
$$

letting $n \rightarrow \infty$, we hence get

$$
f(\sqrt{x y}) \leq \sqrt{f(x) f(y)}, \quad x, y>1,
$$

that is, $f$ is Jensen geometrically convex in $(1, \infty)$ (or, equivalently, the function $\log \circ f \circ \exp$ is Jensen convex in the interval $(0, \infty))$. 
If condition (ii) is satisfied, then

$$
\left[f\left(\left(\frac{x+y}{2}\right)^{p_{n}}\right)\right]^{q_{n}} \leq \frac{\left[f\left(x^{p_{n}}\right)\right]^{q_{n}}+\left[f\left(y^{p_{n}}\right)\right]^{q_{n}}}{2}, \quad x, y>1 ; n \in \mathbb{N} .
$$

Replacing here $x$ and $y$ by $x^{1 / p_{n}}$ and $y^{1 / p_{n}}$, respectively, we get

$$
\begin{aligned}
f\left(\left(\frac{x^{1 / p_{n}}+y^{1 / p_{n}}}{2}\right)^{p_{n}}\right) & \\
\leq & \left(\frac{[f(x)]^{q_{n}}+[f(y)]^{q_{n}}}{2}\right)^{1 / q_{n}}, \quad x, y>1 ; n \in \mathbb{N} .
\end{aligned}
$$

Since $\lim _{n \rightarrow \infty} p_{n}=\infty, \lim _{n \rightarrow \infty} q_{n}=0$, letting here $n \rightarrow \infty$ and applying (3.1), we obtain

$$
f(\sqrt{x y}) \leq \sqrt{f(x) f(y)}, \quad x, y>1,
$$

that is, $f$ is Jensen geometrically convex in $(1, \infty)$.

By the assumption there are a set $A \subset(0, \infty)$ of positive Lebesgue measure or of the second Baire category and $M>0$ such that

$$
f(x) \leq M, \quad x \in A .
$$

Since there is $n \in \mathbb{N}$ such that $A \cap(0, \infty)$ is also of positive Lebesgue measure or of the second Baire category, we may assume that $A$ is bounded, that is, $m:=\sup A<\infty$. For sufficiently large $k$, we have $k+A \subset(a, \infty)$ and $k+A$ is of positive Lebesgue measure or of the second Baire category. From (1), by induction, we have

$$
f(x+k)=x(x+1) \cdot \ldots \cdot(x+k-1) f(x), \quad x>0 .
$$

Hence,

$$
f(x+k) \leq m(m+1) \cdot \ldots \cdot(m+k-1) M, \quad x \in(k+A),
$$

that is, $f$ is bounded from above on the set $k+A \subset(a, \infty)$. By the theorem of Ostrowski and the theorem of Mehdi (cf., Kuczma [6, page $210]$, the function $\log \circ f \circ \exp$ is convex in the interval $(\log a, \infty)$, that is,

$\log \circ f \circ \exp (t u+(1-t) v) \leq t \log \circ f \circ \exp (u)+(1-t) \log \circ f \circ \exp (v)$, for all $t \in(0,1)$ and $u, v \in(\log a, \infty)$, or equivalently,

$$
f\left(x^{t} y^{1-t}\right) \leq[f(x)]^{t}[f(y)]^{1-t}, \quad t \in(0,1), x, y \in(a, \infty) .
$$


Thus, $f$ is geometrically convex, and the result follows from [4].

Remark 3.2. The function $f$ satisfies the assumed regularity conditions in Theorem 3.1 if it is Lebesgue measurable or continuous at a point (cf., Kuczma [6]).

Remark 3.3. The assumption of the convexity. From Theorem 3.1, we immediately obtain the following:

Corollary 3.4. Assume that $f:(0, \infty) \rightarrow(0, \infty)$ is bounded from above on a set of positive Lebesgue measure or on a set of the second Baire category and satisfies (1). If, for every positive integer $n$, the function $x \mapsto\left[f\left(x^{n}\right)\right]^{1 / n}$ is Jensen convex in the interval $(1, \infty)$, then $f$ is the Euler gamma function.

\section{REFERENCES}

1. H. Alzer and J. Matkowski, A convexity property and a new characterization of Euler's gamma function, Arch. Math. 100 (2013), 131-137.

2. E. Artin, Einführung in die Theorie der Gammafunktion, Teubner, Leipzig, 1931. The gamma function, M. Butler, Holt, Rinehart and Winston, San Francisco, 1964 (in English).

3. H. Bohr and J. Mollerup, Laerebog i Matematisk Analyse, III, Jul. Gjellerups Forlag, Copenhagen, 1922.

4. D. Gronau and J. Matkowski, Geometrical convexity and generalization of the Bohr-Mollerup theorem on the gamma function, Math. Pannonica 4 (1993), 153-160.

5. , Another characterization of the gamma function, Publ. Math. Debrecen 63 (2003), 105-113.

6. M. Kuczma, An introduction to the theory of functional equations and inequalities, Polish Sci. Editors Silesian University, Warszawa, Poland, 1985.

7. M.R. Mehdi, On convex functions, J. Lond. Math. Soc. 39 (1964), 321-326.

8. A. Ostrowski, Zur Theorie der konvexen Funktionen, Math. Helv. 1 (1929), $157-159$.

University of Zielona Góra Faculty of Mathematics, Computer Science and Econometrics, Szafrana 4a, PL-65-516 Zielona Góra, Poland

Email address: J.Matkowski@wmie.uz.zgora.pl 